\title{
No overall change in the rate of weight gain after switching to an integrase-inhibitor in virologically suppressed adults with HIV James E. Burns ${ }^{a, b}$, Oliver T. Stirrup ${ }^{a}$, David Dunn ${ }^{a, c}$, Iain Runcie-Unger ${ }^{a, b}$, Ana Milinkovic ${ }^{a, d}$, Sophie Candfield ${ }^{b}$, Hinal Lukha $^{a, b}$, Abigail Severn ${ }^{a, b}$, Laura Waters ${ }^{b}$, Simon Edwardss, Richard Gilson $^{\mathrm{a}, \mathrm{b}}$ and Sarah L. Pett ${ }^{\mathrm{a}, \mathrm{b}, \mathrm{c}, \mathrm{e}}$
}

\begin{abstract}
Objective: Excessive weight gain has been reported with integrase strand transfer inhibitors (INSTIs). We evaluated weight changes in virologically suppressed adults with HIV who switched from non-INSTI regimens to raltegravir (RAL)-containing or dolutegravir (DTG)-containing antiretroviral therapy.

Design: Retrospective single-centre cohort.

Methods: Adults who switched to RAL or DTG before or between January 2015 and October 2017 were identified. Virologically suppressed, treatment-experienced $(\geq 2$ years) individuals, at least 6 months on INSTI, with weight measurements 2 years or less pre and postswitch were included. Our analysis used a random effects model with linear slope pre and post-INSTI with adjustment for age, sex, ethnicity, preswitch-regimen (protease inhibitor vs. nonprotease inhibitor), and RAL vs. DTG use.
\end{abstract}

Results: A total of 378 individuals, $81.2 \%$ male, $70.1 \%$ white ethnicity, median age of 49 years, median of four weight measurements per participant, and median weight and BMl at switch of $76.6 \mathrm{~kg}$ and $25.3 \mathrm{~kg} / \mathrm{m}^{2}$, respectively, were included. Weight increased by an average of $0.63 \mathrm{~kg} /$ year $(95 \%$ confidence interval $0.17-1.09)$ preswitch with no overall change in rate of weight gain postswitch $[+0.05 \mathrm{~kg} /$ year $(-0.61-0.71$, $P=0.88)$ ]. In our adjusted model, a transition from minimal weight change to weight gain postswitch was isolated to older individuals though this lacked statistical significance [e.g., $+1.59 \mathrm{~kg} /$ year $(-0.26-3.45)$ if aged 65 years]. Our findings did not differ by sex, ethnicity, preswitch regimen, or RAL vs. DTG. Similar results were seen for BMI and after adjusting for fixed nucleoside/nucleotide reverse transcriptase inhibitor backbone. Conclusion: We found no clear evidence of an overall increase in rate of weight gain following switch to INSTI in virologically suppressed individuals.

Copyright @ 2019 The Author(s). Published by Wolters Kluwer Health, Inc.

AIDS 2020, 34:109-114

Keywords: antiretroviral therapy, drug switch, integrase inhibitors, weight gain

\footnotetext{
${ }^{\mathrm{a}}$ Centre for Clinical Research in Infection and Sexual Health, Institute for Global Health, University College London, ${ }^{\mathrm{b}}$ Central and North West London NHS Foundation Trust, Mortimer Market Centre, 'Institute of Clinical Trials and Methodology, University College London, ${ }^{\mathrm{d}}$ Chelsea and Westminster Hospital NHS Foundation Trust, London, UK, and ${ }^{\mathrm{e}}$ Kirby Institute, University of New South Wales, Sydney, New South Wales, Australia.

Correspondence to James E. Burns, MBBS, MSc, Centre for Clinical Research in Infection and Sexual Health, Institute for Global Health, University College London, Mortimer Market Centre, Capper Street, London WC1E 6JB, UK.

E-mail: james.burns@ucl.ac.uk
}

Received: 21 June 2019; revised: 20 August 2019; accepted: 22 August 2019. 


\section{Introduction}

Integrase strand transfer inhibitors (INSTI) are recommended as part of antiretroviral treatment (ART) in multiple guidelines [1-4]. Raltegravir (RAL) and dolutegravir (DTG) are widely prescribed in the UK because of their favourable efficacy, lipid, and drug interaction profiles, and tolerability [5-9]. However, there are concerns about excessive weight gain, though findings are inconsistent and uncertainty persists around whether there is a specific effect attributable to INSTIs.

Some observational cohorts showed weight gain (average $\approx 3 \mathrm{~kg}$ over $48-78$ weeks) after switch to INSTI $[10,11]$. In contrast, recent switch studies showed a majority experiencing no weight gain [12], small gains in weight $(0.2 \mathrm{~kg} /$ year $)$ [13], or BMI changes less than $2 \mathrm{~kg} / \mathrm{m}^{2}$ postswitch [14]. In NEAT-022, participants on proteaseinhibitor-based ART were randomized to immediateswitch or deferred-switch to DTG. Both groups experienced weight gain though this, was less than $1.25 \mathrm{~kg}$ over 96 weeks [15].

We performed a retrospective analysis on an adult, virologically suppressed HIV cohort in London, UK, to assess the impact on weight of switching to a DTGcontaining or RAL-containing regimen.

\section{Methods}

Data extraction from clinic records identified adults with HIV who received DTG or RAL between 1 January 2015 and 1 October 2017. The inclusion criteria were: any ART combination for at least 2 years pre-INSTI, virologically suppressed $(<50$ copies $/ \mathrm{ml}$ during the 2 years preswitch; nonsustained viral load 'blips' of $>50$, $<200$ copies/ml were allowed), switch to DTG/RAL during or before the period above, on DTG/RAL for at least 6 months, at least one weight measurement before or at switch, and at least one weight measurement postswitch. Weight/height measurements were performed in HIV outpatient clinics with standard operating procedures that include regular device calibration and were included up to 2 years pre and postswitch. Weights and viral loads within 1 year of ART initiation, individuals with prior elvitegravir (EVG) exposure, and weights recorded after postswitch viral failures (viral load $>200$ copies $/ \mathrm{ml}$ ) were excluded. In cases of multiple RAL and/or DTG regimens only data pertaining to the first were included.

\section{Statistical analysis}

Weight was analysed using a random effects model with linear slope before and after switch to INSTI. A threshold of weight gain (e.g. $10 \%$ gain) was not chosen due to heterogeneity in the timings of weight measurements.
Person-level correlated random effect terms were included for intercept and pre and postswitch slopes. The model was defined in terms of averages for weight at switch, annual rate of weight gain preswitch, and change in rate of weight gain postswitch. This was repeated in a separate model for BMI. Each model component had parameters estimated to evaluate associations with age, sex, ethnicity (Black African or other nonwhite/ unknown vs. white), and pre and postswitch regimens (protease-inhibitor vs. nonprotease-inhibitor and DTG vs. RAL, respectively). Associations between age at switch and INSTI with each weight or BMI trajectory characteristic were modelled on a continuous scale using natural cubic splines with knots at 29, 45, 52, and 70 years (2.5th, 33rd, 66th, and 97.5th centiles). Marginal $P$ values were calculated for each component of the models using overall $F$ tests for sets of spline parameters. Models were fitted using the nlme package [16] in $R$ [17].

\section{Results}

The data extraction yielded 1228 ART-experienced individuals who switched to DTG or RAL. We excluded those with: no recorded weights $(n=134)$, insufficient recorded weights $(n=375)$, less than 2 years pre-INSTI ART use $(n=45)$, prior EVG exposure $(n=6)$, switched from nonprotease-inhibitor to protease-inhibitor regimen less than 2 years pre-INSTI $(n=3)$, or documented adherence issues, treatment breaks, or less than 6 months on INSTI $(n=211) .76$ did not meet the virological suppression criteria. The final sample for analysis was 378 participants, with 1726 weight observations between 2012 and 2018, a median of 4 [interquartile range (IQR) 3-6] weights per participant, an average of 9.5 years (IQR 5.8-14.1) from ART initiation to INSTI, and an average of 1.26 years (IQR $0.76-1.68$ ) between switch date and last included weight.

The cohort was predominantly white men $(n=251$, $66.4 \%$ ); median age at switch was 49 years (IQR 43-55), 248 (65.6\%) received RAL vs. 130 (34.4\%) DTG, and median weight and BMI at switch was $76.6 \mathrm{~kg}$ (IQR $70.1-84.4$ ) and $25.3 \mathrm{~kg} / \mathrm{m}^{2}$ (IQR 23.1-27.7), respectively (refer to Table, Supplementary Digital Content 1, http://links.lww.com/QAD/B539, which demonstrates cohort demographics). A mixed effects model unadjusted for demographic or treatment characteristics showed a postswitch weight gain of $0.68 \mathrm{~kg} /$ year [ $95 \%$ confidence interval (CI) 0.33-1.00]; however, this was near-identical to weight gain preswitch $(0.63 \mathrm{~kg} /$ year, $95 \%$ CI $0.17-$ 1.09), with no evidence of a substantial change in the rate of weight gain postswitch $(+0.05 \mathrm{~kg} /$ year, $95 \% \mathrm{CI}$ $-0.61-0.71, P=0.88)$.

Average weights and BMIs by age at switch for the adjusted models are shown in Fig. 1a and e, respectively. 
(a)

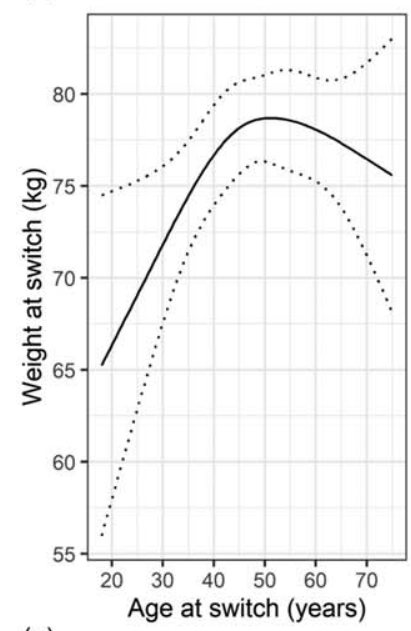

(e)

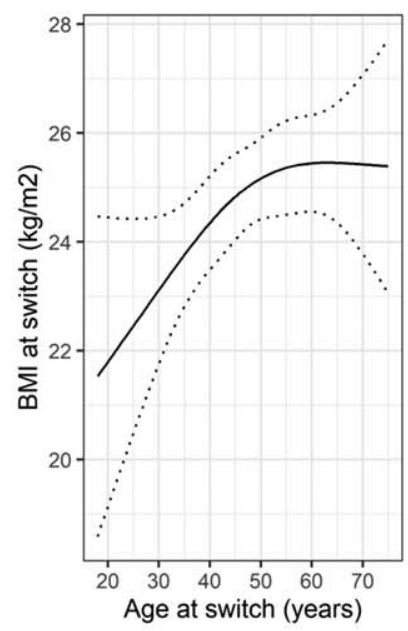

(b)

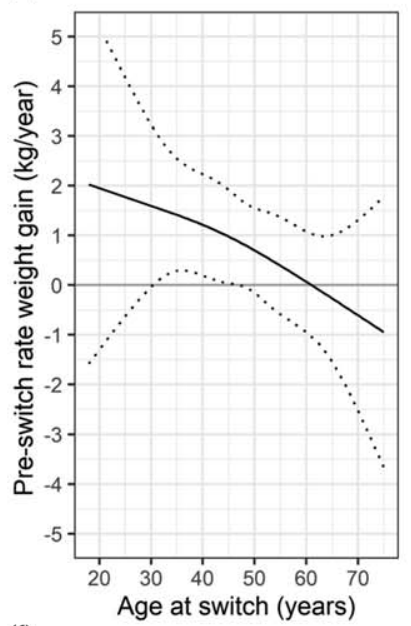

(f)

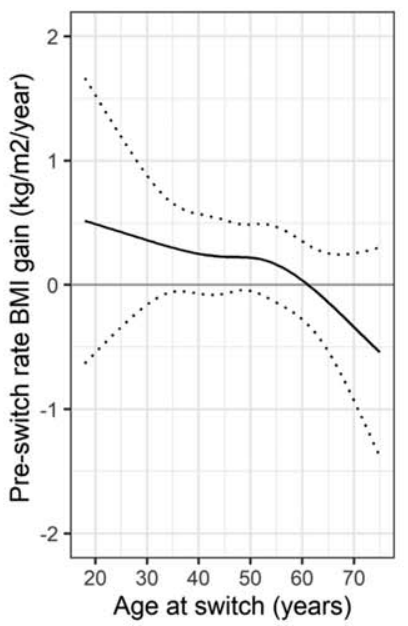

(c)

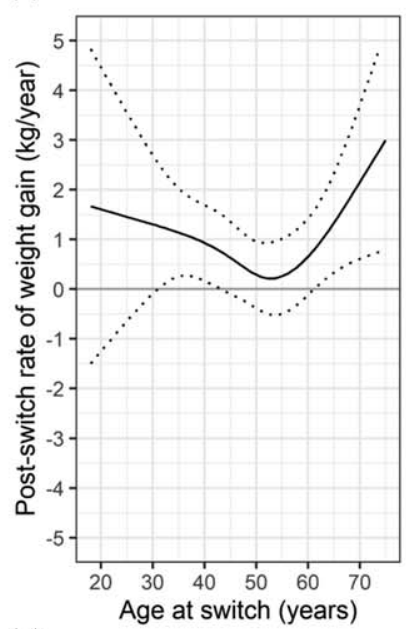

(g)

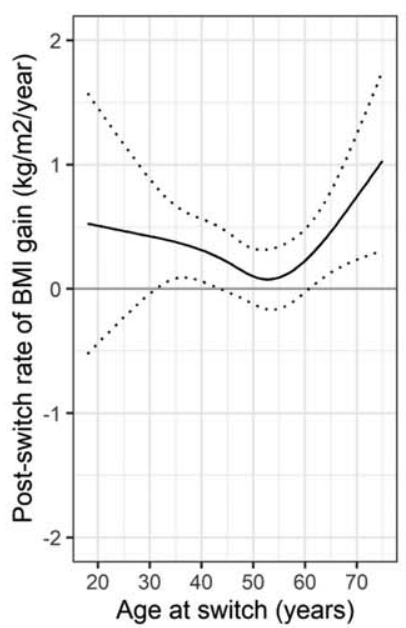

(d)

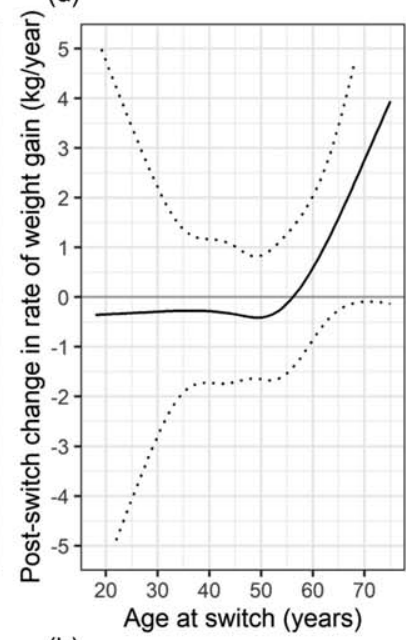

(h)

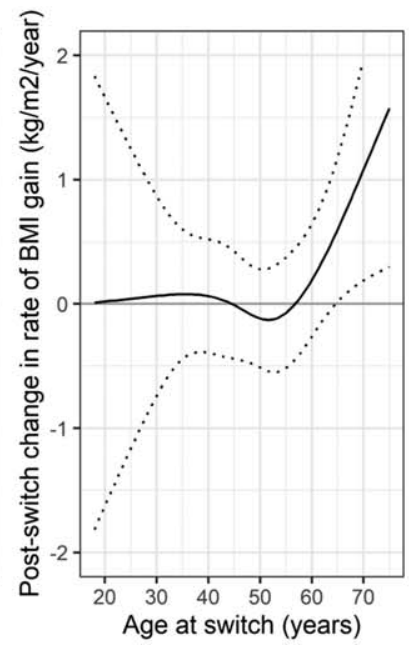

Fig. 1. Estimates of average (a) weight at switch to integrase strand transfer inhibitor regimen, (b) preswitch rate of weight gain and (c) postswitch rate of weight gain, (d) postswitch change in rate of weight gain in relation to age at switch, (e) BMI at switch to integrase strand transfer inhibitor regimen, (f) preswitch rate of BMI gain, (g) postswitch rate of BMI gain, and (h) postswitch change in rate of BMI gain, in relation to age at switch. These estimates are derived from models with adjustment for the demographic and treatment characteristics listed in Table 1. The plots relate to a white male individual switching from a nonprotease inhibitor to a raltegravir-based regimen. Dotted lines show pointwise $95 \%$ confidence interval. Plot (c) represents a summation of the functions in (b) and (d), and ( $g$ ) is a summation of ( $f$ ) and (h).

We estimated individuals aged less than 50 years had an average preswitch rate of weight gain of more than $0.5 \mathrm{~kg} /$ year, whereas those aged more than 60 years predominantly had static weights or a weight loss of less than $1 \mathrm{~kg}$ /year (Fig. 1b, $P=0.09$; spline vs. no preswitch weight gain). Overall, we did not find strong evidence for any postswitch change in the rate of weight gain. We did observe some suggestion of an increased rate of weight gain postswitch in older individuals [e.g. $+1.59 \mathrm{~kg} /$ year (95\% CI $-0.26-3.45)$ if aged 65 years] though this failed to achieve statistical significance (Fig. $1 \mathrm{~d}, P$ value $=0.35$, spline vs. no postswitch change). There were no changes between pre and postswitch rates of weight gain when analysed by sex, ethnicity, and pre or postswitch regimen (protease-inhibitor vs. nonprotease-inhibitor and DTG vs. RAL, respectively) (Table 1). There was substantial variation in the subject-specific rates of pre and postswitch weight gain [SD values $(95 \% \mathrm{CI})$ for the random effect terms of $2.86(2.43-3.36)$ and 2.21 (1.88$2.59) \mathrm{kg} /$ year, respectively].

The analysis using BMI gave similar results (Fig. 1e-h, Table 1). Although there appears to be an indication of a postswitch increase in rate of change of BMI in those aged more than 65 years based on the pointwise 95\% CI (Fig. 1h), the overall relationship between age and postswitch change was nonsignificant $(P=0.20$, spline vs. no postswitch change). 
Table 1. Associations between demographic/treatment characteristics and weight/BMI trajectories.

\begin{tabular}{|c|c|c|c|c|c|c|c|}
\hline & & $\begin{array}{l}\text { Weight or } \\
\text { BMI at switch }\end{array}$ & & $\begin{array}{l}\text { Preswitch rate of weight } \\
\text { or BMI gain (per year) }\end{array}$ & & $\begin{array}{l}\text { Postswitch change in rate of } \\
\text { weight or BMI gain (per year) }\end{array}$ & \\
\hline & $n(\%)$ & $\Delta$ Est. $(95 \% \mathrm{Cl})$ & $P$ value & $\Delta$ Est. $(95 \% \mathrm{Cl})$ & $P$ value & $\Delta$ Est. $(95 \% \mathrm{Cl})$ & $P$ value \\
\hline \multicolumn{8}{|l|}{ Weight results (kg) } \\
\hline \multicolumn{8}{|l|}{ Sex } \\
\hline Men & $307(81.2)$ & 0 [Reference] & & 0 [Reference] & & 0 [Reference] & \\
\hline Women & $71(18.8)$ & $-6.16(-10.35$ to -1.98$)$ & $<0.01$ & $-0.14(-1.60-1.32)$ & 0.85 & $-0.14(-2.3-2.02)$ & 0.90 \\
\hline \multicolumn{8}{|c|}{ 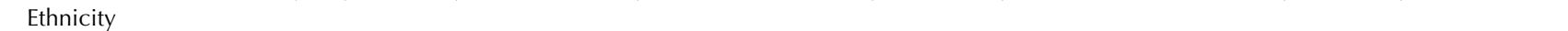 } \\
\hline White & $265(70.1)$ & 0 [Reference] & & 0 [Reference] & & 0 [Reference] & \\
\hline Black African & $67(17.7)$ & $5.04(0.67-9.41)$ & 0.01 & $-0.23(-1.74-1.27)$ & 0.76 & $0.82(-1.38-3.03)$ & 0.47 \\
\hline Other nonwhite & $46(12.2)$ & $3.92(-0.22-8.07)$ & 0.07 & $-0.53(-2.06-1.00)$ & 0.50 & $1.34(-0.88-3.56)$ & 0.24 \\
\hline \multicolumn{8}{|l|}{ Preswitch } \\
\hline Non-PI & $185(48.9)$ & 0 [Reference] & & 0 [Reference] & & 0 [Reference] & \\
\hline PI & $193(51.1)$ & $0.80(-1.86-3.46)$ & 0.56 & $-0.15(-1.12-0.82)$ & 0.76 & $0.15(-1.24-1.54)$ & 0.83 \\
\hline \multicolumn{8}{|l|}{ Postswitch } \\
\hline RAL & $248(65.6)$ & 0 [Reference] & & 0 [Reference] & & 0 [Reference] & \\
\hline DTG & $130(34.4)$ & $0.46(-2.35-3.27)$ & 0.75 & $0.37(-0.67-1.41)$ & 0.49 & $-0.67(-2.14-0.81)$ & 0.38 \\
\hline \multicolumn{8}{|l|}{ BMI results $\left(\mathrm{kg} / \mathrm{m}^{2}\right)$} \\
\hline \multicolumn{8}{|l|}{ Sex } \\
\hline Men & $304(81.7)$ & 0 [Reference] & & 0 [Reference] & & 0 [Reference] & \\
\hline Women & $68(18.3)$ & $2.26(0.93-3.58)$ & $<0.01$ & $0.26(-0.20-0.72)$ & 0.27 & $-0.37(-1.06-0.32)$ & 0.29 \\
\hline \multicolumn{8}{|l|}{ Ethnicity } \\
\hline White & $262(70.4)$ & 0 [Reference] & & 0 [Reference] & & 0 [Reference] & \\
\hline Black African & 65 (17.5) & $2.13(0.75-3.51)$ & $<0.01$ & $-0.16(-0.63-0.31)$ & 0.51 & $0.40(-0.30-1.09)$ & 0.26 \\
\hline Other nonwhite & $45(12.5)$ & $1.59(0.28-2.90)$ & 0.02 & $0.09(-0.39-0.57)$ & 0.71 & $0.13(-0.57-0.84)$ & 0.71 \\
\hline \multicolumn{8}{|l|}{ Preswitch } \\
\hline Non-PI & $182(48.9)$ & 0 [Reference] & & 0 [Reference] & & 0 [Reference] & \\
\hline $\mathrm{PI}$ & $190(51.1)$ & $0.54(-0.30-1.38)$ & 0.21 & $0.02(-0.29-0.32)$ & 0.91 & $-0.06(-0.50-0.39)$ & 0.80 \\
\hline \multicolumn{8}{|l|}{ Postswitch } \\
\hline RAL & $244(65.6)$ & 0 [Reference] & & 0 [Reference] & & 0 [Reference] & \\
\hline DTG & $128(34.4)$ & $-0.02(-0.91-0.87)$ & 0.97 & $0.05(-0.28-0.37)$ & 0.78 & $-0.11(-0.57-0.36)$ & 0.66 \\
\hline
\end{tabular}

Associations between demographic/treatment characteristics and both average weight and BMI trajectories during the 2 years before and after switch to a RAL or DTG-based INSTI regimen. Adjusted for age at switch and the other variables listed in the table. Estimates are expressed relative $(\Delta)$ to the average value at any given age (Fig. 1). Cl, confidence interval; DTG, dolutegravir; PI, protease inhibitor; RAL, raltegravir.

We also considered depression $(n=113,29.9 \%)$, high cholesterol/lipids $(n=57,15.1 \%)$, and renal impairment $(n=37,9.8 \%)$ as predictive factors in our models. High cholesterol/lipids was only associated with higher weight at switch $[+4.1 \mathrm{~kg}(95 \% \mathrm{CI} 0.6-7.7), P$ value $=0.02]$. No associations were seen between depression or renal impairment and weight change. We were unable to consider other comorbidities due to low numbers. CD $4^{+}$ cell count was not included in our models due to missing values, however, $96 \%(n=238 / 247)$ had more than 300 cells/ $\mu$ l [median 640 (IQR 500-790) cells/ $\mu$ l] preswitch. A separate model adjusting for fixed nucleoside/ nucleotide reverse transcriptase inhibitor backbone pre and post switch again observed no overall significant change in rate of weight gain (refer to text/figure/table, Supplementary Digital Content 2, http://links.lww. com/QAD/B539).

\section{Discussion}

We did not find an overall increase in rate of weight or BMI gain following switch to DTG or RAL. This contrasts with some previous studies [11-14], though our CIs do not exclude a rate increase of less than $1 \mathrm{~kg} /$ year. We observed no difference between RAL and DTG, again contrasting reports of greater weight gain in patients switching to DTG, although the differences were small $(<1 \mathrm{~kg} /$ year $)[13,14]$. The observed increase in rate of weight gain for those more than 55 years did not reach statistical significance, although our study was underpowered to detect changes within this age group. Of note, many of our cohort were overweight at baseline and their preswitch trajectory of gradual weight gain continued postswitch. Even small annual increases in weight, when considered cumulatively, could result in cardiovascular and/or metabolic consequences.

A major limitation of our study is the amount of missing weight data. Weights/BMI may be recorded inconsistently in routine clinical care. The British HIV Association guidelines only recommend weight/BMI measurement after initial HIV assessment if evaluating cardiovascular or bone fracture risk, if weight change is a symptom, and annually for those with chronic kidney disease or an increased risk of/established cardiovascular disease [18]. Increased weight monitoring in response to 
patient reporting could create an upward bias in estimates of rate of weight gain. Similarly, we focused on long-term weight gain by excluding individuals on DTG/RAL less than 6 months which may have removed those who experienced very rapid gains. The predominance of white men in our cohort limits generalizing our findings to women and Black African/other nonwhite individuals who may be more susceptible to weight gain [12$14,19,20]$.

We found no association between weight change and depression, which has been reported previously [12]. Low rates of comorbidities such as type 2 diabetes (T2DM) prohibited us from exploring other potential predictive factors. This may be due to under-recording of comorbidities. We lacked data on concurrent medications because they are largely prescribed in primary care and HIV clinic records may be inaccurate. We were also unable to assess lifestyle factors which could impact weight changes.

Small weight gains have been associated with tenofovir alafenamide (TAF) vs. tenofovir disoproxil fumarate (TDF) in the DISCOVER preexposure prophylaxis trial $(+1.1 \mathrm{~kg})$ [21] and a German cohort [22]. However, larger weight gains (mean increase $6.4 \mathrm{~kg}$ over 48 weeks) have been reported recently in naïve patients on TAF + DTG-containing regimens compared with TDF + DTG and TDF + efavirenz-600 $\mathrm{mg}$ in the ADVANCE trial [20]. This suggests TAF may be implicated in the increased weight gain. Due to low use of TAF $(n=11)$, we were unable to evaluate any possible association. Observations of weight gain with other INSTIs are also mixed, that is, EVG $[23,24]$, bictegravir [25,26], and cabotegravir [27].

Assessing factors influencing weight gain remains challenging, evidenced by the high level of subjectspecific variation in rates of weight gain. It is difficult to differentiate potential INSTI effects on weight from weight gain seen in patients due to successful/well tolerated ART, environmental factors, and comorbidities also affecting individuals not living with HIV. National Health Service 2017 data shows that 64\% (63-66) of UK adults are overweight (BMI $25-30 \mathrm{~kg} / \mathrm{m}^{2}$ ) or obese $\left(\mathrm{BMI}>30 \mathrm{~kg} / \mathrm{m}^{2}\right)$, a $3 \%$ increase since 2016 [28]. The Organisation for Economic Co-operation and Development forecasts up to $35 \%$ of UK adults being obese by 2030 [29]. In addition, the proportion of overweight/ obese UK adults is highest in men aged $45-74$ years (78\%) and women aged 65-74 years (73\%) [28]. Weight gain postswitch in those more than 55 years may reflect this general trend.

The lack of consensus around whether observed weight gains can be attributed to INSTIs, or to general environmental/lifestyle factors, highlights the importance of reliably monitoring weight in people living with HIV
(PLWH). The nature of weight gain in terms of fat vs. lean mass may also be important and, as demonstrated in ADVANCE, may differ by sex [20]. Ongoing weight gain in virologically suppressed PLWH, especially central weight gain and its sequelae - including T2DM, increased cardiovascular risk, and nonalcoholic fatty liver disease are research priorities, as are strategies to maintain healthy BMIs for this population. The discordance amongst studies highlights a need to remain critical of whether weight gain observed with INSTIs is truly a widespread class effect warranting action, or whether lifestyle and population factors may have greater influence and thus should be given precedence.

\section{Acknowledgements}

Authors' contributions: J.E.B., S.L.P., D.D., O.T.S., and A.M. developed the research question and study design following previous design work and data collection conducted by S.C., A.M., H.L., S.E., and A.S. I.R. performed the electronic data extraction. J.E.B. completed further electronic clinical record reviews and data collection, data cleaning, and drafted the initial article text. O.T.S. performed the statistical analyses and contributed towards the initial draft text of the article. All authors were involved in the final interpretation of the results and final text of the article.

Participating investigator: The authors would also like to acknowledge the assistance of Stephen Ogunnaike with part of the data extraction.

J.E.B.: supported by the National Institute for Health Research Comprehensive Research Network. S.L.P.: portion of salary comes through Medical Research Council core funding (MR_UU_12023).

\section{Conflicts of interest}

A.M.: speaker honoraria for MSD, Janssen, and Gilead Sciences. Received support for conference attendance from Gilead Sciences and Janssen. L.W.: received speaker fees, advisory fees, and support for conference attendance from Gilead Sciences, MSD, ViiV, Janssen, Mylan, and Cipla. Involved in research sponsored by Gilead Sciences and Janssen. For the remaining authors none were declared.

\section{References}

1. European AIDS Clinical Society. European AIDS Clinical Society (EACS) guidelines. London: EACS; 2018, 9.1.

2. World Health Organization. Updated recommendations on first-line and second-line antiretroviral regimens and postexposure prophylaxis and recommendations on early infant diagnosis of HIV. Geneva, Switzerland: WHO; 2018. 
3. Saag MS, Benson CA, Gandhi RT, Hoy JF, Landovitz RJ, Mugavero $\mathrm{MJ}$, et al. Antiretroviral drugs for treatment and prevention of HIV infection in adults: 2018 recommendations of the International Antiviral Society - USA Panel. JAMA 2018; 320:379-396.

4. British HIV Association. British HIV Association guidelines for the treatment of HIV-1-positive adults with antiretroviral therapy (interim update). London: BHIVA; 2016.

5. Iwamoto $M$, Wenning LA, Petry AS, Laethem M, De Smet $M$, Kost JT, et al. Safety, tolerability, and pharmacokinetics of raltegravir after single and multiple doses in healthy subjects. Clin Pharmacol Ther 2008; 83:293-299.

6. Mondi A, Cozzi-Lepri A, Tavelli A, Rusconi S, Vichi F, Ceccherini-Silberstein $\mathrm{F}$, et al. Effectiveness of dolutegravir-based regimens as either first-line or switch antiretroviral therapy: data from the Icona cohort. J Int AIDS Soc 2019; 22:e25227.

7. Naumann U, Moll A, Schleehauf D, Lutz T, Schmidt W, Jaeger $\mathrm{H}$, et al. Similar efficacy and tolerability of raltegravir-based antiretroviral therapy in HIV-infected patients, irrespective of age group, burden of comorbidities and concomitant medication: real-life analysis of the German 'WIP' cohort. Int / STD AIDS 2017; 28:893-901.

8. Quercia R, Roberts J, Martin-Carpenter L, Zala C. Comparative changes of lipid levels in treatment-naive, HIV-1-infected adults treated with dolutegravir vs. efavirenz, raltegravir, and ritonavir-boosted darunavir-based regimens over 48 weeks. Clin Drug Investig 2015; 35:211-219.

9. McComsey GA, Moser C, Currier J, Ribaudo HJ, Paczuski P, Dubé MP, et al. Body composition changes after initiation of raltegravir or protease inhibitors: ACTG A5260s. Clin Infect Dis 2016; 62:853-862.

10. Menard A, Meddeb L, Tissot-Dupont H, Ravaux I, Dhiver C, Mokhtari $S$, et al. Dolutegravir and weight gain: an unexpected bothering side effect? AIDS 2017; 31:1499-1500.

11. Norwood J, Turner M, Bofill C, Rebeiro P, Shepherd B, Bebawy S, et al. Brief report: weight gain in persons with HIV switched from efavirenz-based to integrase strand transfer inhibitor-based regimens. J Acquir Immune Defic Syndr 2017; 76:527-531.

12. Mocomsey GA, Eron II, Santiago S, Mounzer K, Moyle G, Vanig $\mathrm{T}$, et al. Weight gain during treatment among 3,468 treatmentexperienced adults with HIV. [Abstract 671] Conference on Retroviruses and Opportunistic Infections (CROI); 4-7 March 2019.

13. Lake JE, Wu K, Erlandson KM, Bares SH, Debroy P, Godfrey C, et al. Risk factors for excess weight gain following switch to integrase inhibitor-based ART. [Abstract 669] Conference on Retroviruses and Opportunisitc Infections (CROI); 4-7 March 2019.

14. Palella FJ, Rayeed N, Li J, Ward D, Fuhrer J, Purinton S, et al. Weight gain among virally suppressed persons who switch to INSTI-based ART. [Abstract 674] Conference on Retroviruses and Opportunistic Infections (CROI); 4-7 March 2019.

15. Waters L, Assoumou L, Rusconi S, Domingo P, Gompels M, De Wit S, et al. Switch to dolutegravir from a boosted protease inhibitor associated with a significant weight gain over 48 weeks in NEAT-022, a randomised 96-week trial. [Abstract P102] HIV Glasgow; 28-31 October 2018.
16. Pinheiro J, Bates D, DebRoy S, Sarkar D, R Core Team. nlme: linear and nonlinear mixed effects models. $\mathrm{R}$ package version 3.1-137 2018.

17. R Core Team. R: a language and environment for statistical computing. Vienna, Austria: R Foundation for Statistical Computing; 2013.

18. British HIV Association. BHIVA guidelines for the routine investigation and monitoring of adult HIV-1-positive individuals 2016 (2019 interim update). United Kingdom: BHIVA; 2019.

19. Bedimo R, Adams-Huet B, Xilong L, Lake JE, Taylor B, Tebas P, et al. Integrase inhibitors-based HAART is associated with greater BMI gains in Blacks and Hispanics. [Abstract 549] ID Week; 2-7 October 2018.

20. Venter WDF, Moorhouse M, Sokhela S, Fairlie L, Mashabane N, Masenya $M$, et al. Dolutegravir plus two different prodrugs of tenofovir to treat HIV. N Engl J Med 2019; 381:803-815.

21. Hare CB, Coll J, Ruane $\mathrm{P}$, Molina, J-M, Mayer $\mathrm{KH}$, Jessen $\mathrm{H}$, et al. The phase 3 DISCOVER study: Daily F/TAF or F/TDF for HIV preexposure prophylaxis. [Abstract 104] Conference on Retroviruses and Opportunistic Infections (CROI); 4-7 March 2019.

22. Gomez M, Seybold U, Roider J, Harter G, Bogner JR. A retrospective analysis of weight changes in HIV-positive patients switching from a tenofovir disoproxil fumarate (TDF)- to a tenofovir alafenamide fumarate (TAF)-containing treatment regimen in one German university hospital in 2015-2017. Infection 2019; 47:95-102.

23. Bedimo R, Li X, Adams-Huet B, Lake JE, Taylor BS, Kim D, et al. Differential BMI changes following PI- and INSTI-based ART initiation by sex and race. [Abstract 675] Conference on Retroviruses and Opportunistic Infections (CROI); 4-7 March 2019.

24. Bourgi K, Rebeiro PF, Turner M, Castilho JL, Hulgan T, Raffanti $\mathrm{SP}$, et al. Greater weight gain in treatment-naive persons starting dolutegravir-based antiretroviral therapy. Clin Infect Dis 2019[Epub ahead of print].

25. Wohl DA, Yazdanpanah Y, Baumgarten A, Clarke A, Thompson MA, Brinson C, et al. Bictegravir combined with emtricitabine and tenofovir alafenamide versus dolutegravir, abacavir, and lamivudine for initial treatment of HIV-1 infection: week 96 results from a randomised, double-blind, multicentre, phase 3 , noninferiority trial. Lancet HIV 2019; 6:e355-e363.

26. Stellbrink HJ, Arribas JR, Stephens JL, Albrecht H, Sax PE, Maggiolo $F$, et al. Co-formulated bictegravir, emtricitabine, and tenofovir alafenamide versus dolutegravir with emtricitabine and tenofovir alafenamide for initial treatment of HIV-1 infection: week 96 results from a randomised, double-blind, multicentre, phase 3, noninferiority trial. Lancet HIV 2019; 6:e364-e372.

27. Landovitz RJ, Zangeneh SZ, Chau G, Grinsztejn B, Eron IJ Dawood $\mathrm{H}$, et al. Cabotegravir is not associated with weight gain in human immunodeficiency virus-uninfected individuals in HPTN 077. Clin Infect Dis 2019[Epub ahead of print].

28. NHS Digital. Health survey for England 2017 adult overweight and obesity. United Kingdom: NHS Digital; 2017.

29. Organisation for Economic Co-operation and Development. Obesity update 2017. Paris, France: OECD; 2017. 